\title{
A Singular Sturm-Liouville Problem with Limit Circle Endpoints and Eigenparameter Dependent Boundary Conditions
}

\author{
Jinming Cai and Zhaowen Zheng \\ School of Mathematical Sciences, Qufu Normal University, Qufu, Shandong 273165, China \\ Correspondence should be addressed to Zhaowen Zheng; zhwzheng@126.com
}

Received 5 December 2016; Accepted 23 January 2017; Published 21 March 2017

Academic Editor: Chris Goodrich

Copyright ( 2017 Jinming Cai and Zhaowen Zheng. This is an open access article distributed under the Creative Commons Attribution License, which permits unrestricted use, distribution, and reproduction in any medium, provided the original work is properly cited.

\begin{abstract}
In this paper, we investigate a class of discontinuous singular Sturm-Liouville problems with limit circle endpoints and eigenparameter dependent boundary conditions. Operator formulation is constructed and asymptotic formulas for eigenvalues and fundamental solutions are given. Moreover, the completeness of eigenfunctions is discussed.
\end{abstract}

\section{Introduction}

It is well known that many topics in mathematical physics require the investigation of eigenvalues and eigenfunctions of the Sturm-Liouville problems. The theory of regular SturmLiouville problems is well built; since the foundation work of Weyl on limit-point/limit-circle classification [1], the singular Sturm-Liouville problems (see [2-7] for real coefficients and [8] for complex coefficients) and more general Hamiltonian systems (see $[9,10]$ ) are widely researched. Meanwhile, a large number of researchers are interested in the discontinuous Sturm-Liouville problem with inner discontinuous points, since these problems are of wide applications in engineering and mechanics (see [11-25]). Various physics applications of this kind of problems are found, such as oscillation of linear or nonlinear equation (see [26-29]) and heat and mass transfer problems (see [30]).

The regular Sturm-Liouville problems with transmission conditions containing an eigenparameter on one of the boundary conditions have received a lot of attention in research (see [18-22]). Based on these results, some researchers studied the regular Sturm-Liouville problems with eigenparameter on both of the boundary conditions (see [2325]). In these papers, Yang and Wang in [18] considered a Sturm-Liouville problem with discontinuities at two points and eigenparameter dependent boundary condition at one endpoint; they obtained the fundamental solutions and gave the asymptotic formulas of eigenvalues and fundamental solutions. Further, they studied the discontinuous SturmLiouville problem with eigenparameter boundary conditions at two endpoints in [24] and extended the results of [18] to finite discontinuities case. In papers $[19,22,23,25]$, the authors obtained the estimations of eigenvalues and eigenfunctions of the discontinuous Sturm-Liouville problem with one inner point, containing an eigenparameter in the boundary condition. Şen et al. considered the Sturm-Liouville problem with two inner points containing an eigenparameter in the boundary condition and got similar result, respectively (see $[20,21])$. Besides, the authors also discussed the completeness of the eigenfunctions of a regular discontinuous Sturm-Liouville problem in papers [18, 25]. All of them researched the regular Sturm-Liouville problem. However, little is known about the singular Sturm-Liouville problems with limit-circle endpoints.

We will consider the following singular discontinuous Sturm-Liouville problem with two limit-circle endpoints and eigenparameter in the boundary conditions:

$$
\begin{aligned}
L y:=-\left(p(x) y^{\prime}(x)\right)^{\prime}+q(x) y(x)=\lambda y(x), & \\
x & \in(a, \xi) \cup(\xi, b),-\infty<a<\xi<b<\infty,
\end{aligned}
$$

with eigenparameter dependent conditions at the endpoints $a$ and $b$ :

$$
\begin{aligned}
L_{1} y & :=a_{1}[y, u](a)-a_{2}[y, v](a) \\
& =\lambda\left(b_{1}[y, u](a)-b_{2}[y, v](a)\right),
\end{aligned}
$$




$$
\begin{aligned}
L_{2} y & :=c_{1}[y, u](b)-c_{2}[y, v](b) \\
& =\lambda\left(d_{1}[y, u](b)-d_{2}[y, v](b)\right)
\end{aligned}
$$

the transmission conditions at $x=\xi$ are

$$
\begin{aligned}
& L_{3} y:=y(\xi+0)-\delta_{1} y(\xi-0)-\delta_{2} y^{\prime}(\xi-0)=0, \\
& L_{4} y:=y^{\prime}(\xi+0)-\delta_{3} y(\xi-0)-\delta_{4} y^{\prime}(\xi-0)=0,
\end{aligned}
$$

(we assume that not all $\xi_{j}(1 \leq j \leq 4)$ are equal to zero, since, in this case, the boundary value problem (1)-(3) has no transmission conditions), where both $a$ and $b$ are limitcircle points, $u_{1}(x)$ and $v_{1}(x)$ are linearly independent realvalued solutions of equation $-\left(p(x) y^{\prime}(x)\right)^{\prime}+q(x) y(x)=0$ on $(a, \xi)$, and $u_{2}(x)$ and $v_{2}(x)$ are linearly independent realvalued solutions of equation $-\left(p(x) y^{\prime}(x)\right)^{\prime}+q(x) y(x)=0$ on $(\xi, b)$ and satisfy $\left[u_{1}, v_{1}\right](a) \neq 0,\left[u_{2}, v_{2}\right](b) \neq 0$, where $[u, v]=p\left(u \overline{v^{\prime}}-u^{\prime} \bar{v}\right)$ is the sesquilinear form; $p(x)=1 / p_{1}^{2}$ for $x \in(a, \xi), p(x)=1 / p_{2}^{2}$ for $x \in(\xi, b)$, and $\lambda \in \mathbb{C}$ is a spectral parameter; $q(x)$ is a real-valued continuous function on $(a, \xi) \cup(\xi, b)$ and has finite limits $q(\xi \pm 0)=\lim _{x \rightarrow(\xi \pm 0)} q(x)$; $p_{i}, a_{i}, b_{i}, c_{i}, d_{i}(i=1,2)$, and $\delta_{i}(i=1,2,3,4)$ are nonzero real numbers.

For the convenience, we set

$$
\begin{aligned}
& u= \begin{cases}u_{1}, & x \in(a, \xi), \\
u_{2}, & x \in(\xi, b),\end{cases} \\
& v= \begin{cases}v_{1}, & x \in(a, \xi), \\
v_{2}, & x \in(\xi, b) .\end{cases}
\end{aligned}
$$

Moreover, we assume that

$$
\begin{aligned}
& \rho=\left|\begin{array}{ll}
b_{1} & a_{1} \\
b_{2} & a_{2}
\end{array}\right|>0, \\
& \gamma=\left|\begin{array}{ll}
\delta_{1} & \delta_{3} \\
\delta_{2} & \delta_{4}
\end{array}\right|>0, \\
& \theta=\left|\begin{array}{ll}
c_{1} & c_{2} \\
d_{1} & d_{2}
\end{array}\right|>0 .
\end{aligned}
$$

Here, we research a singular Sturm-Liouville problem with two limit-circle endpoints and the parameter $\lambda$ is not only in the equation but also in the boundary conditions. Based on the modified inner product, we define a new self-adjoint operator $A$ such that the eigenvalues of such a problem are coincided with those of $A$. We rebuild its fundamental solutions, get the asymptotic formulas for eigenvalues and eigenfunctions, and also discuss the completeness of its eigenfunctions.

At first, we introduce the following lemmas.

Lemma 1 (the Lagrange identity, see [5]). Let $D(L)=\{y \mid y \in$ $\left.L^{2}[a, b], y^{\prime} \in A C[a, b], L y \in L^{2}[a, b]\right\}$, where, for any interval
$I \subset \mathbb{R}, L^{2}(I)$ denotes the set of functions on $I$ which are square integrable on $I$. For any $y \in D(L), z \in D\left(L^{*}\right)$, one has

$$
\bar{z} L y-y \overline{L^{*} z}=\frac{d}{d x}[y, z](x),
$$

where $L^{*}$ is the adjoint expression of $L$, which is given by $L^{*} z=$ $-\left(\bar{p} z^{\prime}\right)^{\prime}+\bar{q} z$.

Lemma 2 (Green's formula, see [5]). Let $I=[a, b]$; for arbitrary $y \in D(L), z \in D\left(L^{*}\right), D(L)$ and $D\left(L^{*}\right)$ are defined as above; one has

$$
\begin{aligned}
(L y, z)-\left(y, L^{*} z\right) & =\int_{a}^{b}\left(\bar{z} L y-y \overline{L^{*} z}\right) d x \\
& =[y, z](b)-[y, z](a) .
\end{aligned}
$$

Since Ly is of limit-circle type at $x=a$, $\int_{a}^{x_{1}} \bar{u} L y d x, \int_{a}^{x_{1}} y \overline{L^{*} u} d x$ and $[y, u]\left(x_{1}\right)$ exist $\forall x \in\left(a, x_{1}\right)$. So $[y, u](a)=\lim _{x \rightarrow a^{+}}[y, u](x)$ exists. Similarly $[y, v](a)$, $[y, u](b)$ and $[y, v](b)$ exist.

Lemma 3 (see [5]). For any $f, g, \alpha, \beta \in D(L)$, while $D(L)$ is defined in Lemma 1, one has

$$
\left|\begin{array}{ll}
{[f, \alpha]} & {[f, \beta]} \\
{[g, \alpha]} & {[g, \beta]}
\end{array}\right|=[f, \bar{g}][\bar{\alpha}, \beta] .
$$

\section{Operator Formulation}

In this section, we introduce the Hilbert space $H=L^{2}(a, \xi) \oplus$ $L^{2}(\xi, b) \oplus \mathbb{C}^{2}$; the inner product on $H$ is defined by

$$
\begin{aligned}
\langle F, G\rangle= & \gamma p_{1}^{2} \int_{a}^{\xi} f(x) \overline{g(x)} d x+p_{2}^{2} \int_{\xi}^{b} f(x) \overline{g(x)} d x \\
& +\frac{\gamma}{\rho} f_{1} \overline{g_{1}}+\frac{1}{\theta} f_{2} \overline{g_{2}},
\end{aligned}
$$

for $F=\left(f, f_{1}, f_{2}\right), G=\left(g, g_{1}, g_{2}\right) \in H, f, g \in L^{2}((a, \xi) \cup$ $(\xi, b))$. For the convenience, we use the following notations: $H_{1}=L^{2}(a, \xi) \oplus L^{2}(\xi, b)$,

$$
\begin{aligned}
& T_{a}(y)=b_{1}[y, u](a)-b_{2}[y, v](a), \\
& T_{a}^{\prime}(y)=a_{1}[y, u](a)-a_{2}[y, v](a), \\
& T_{b}(y)=d_{1}[y, u](b)-d_{2}[y, v](b), \\
& T_{b}^{\prime}(y)=c_{1}[y, u](b)-c_{2}[y, v](b) .
\end{aligned}
$$

Besides, we introduce the operator $A$ in the Hilbert space $H$ as follows:

$$
D(A)=\left\{F=\left(f(x), f_{1}, f_{2}\right) \in H \mid f(x),\right.
$$

$f^{\prime}(x)$ are absolutely continuous on $(a, \xi)$

$\cup(\xi$,

b) with finite limits $f(\xi \pm 0), f^{\prime}(\xi \pm 0)$ and satisfy $L f$

$$
\begin{aligned}
& \in L^{2}((a, \xi) \cup(\xi, b)), L_{3} f=L_{4} f=0, f_{1}=T_{a}(f), f_{2} \\
& \left.=T_{b}(f)\right\},
\end{aligned}
$$


which acts by the rule

$$
A F=\left(L f, T_{a}^{\prime}(f), T_{b}^{\prime}(f)\right)
$$

with $F=\left(f, T_{a}(f), T_{b}(f)\right) \in D(A)$. Now we can rewrite problem (1)-(4) in the operator form $A F=\lambda F$, for $F=\left(f, T_{a}(f)\right.$, $\left.T_{b}(f)\right) \in D(A)$. Obviously, we have the following lemmas.

Lemma 4. The eigenvalues and eigenfunctions of problem (1)-(4) are corresponding to the eigenvalues and the first component of the corresponding eigenfunctions of operator $A$, respectively.

Lemma 5. The domain $D(A)$ is dense in $H$.

Proof. Let $F=\left(f(x), f_{1}, f_{2}\right) \in H, F \perp D(A)$ and let $C_{0}^{\infty}$ be a functional set which has compact support and can be differential infinitely such that $\vartheta_{1}(x) \in C_{0}^{\infty}(a, \xi), \vartheta_{2}(x) \in C_{0}^{\infty}(\xi, b)$. Since $C_{0}^{\infty} \oplus 0 \oplus 0 \subset D(A)$, for $\forall G=(g(x), 0,0) \in C_{0}^{\infty} \oplus 0 \oplus 0$ is orthogonal to $F$, namely,

$$
\begin{aligned}
\langle F, G\rangle & =\gamma p_{1}^{2} \int_{a}^{\xi} f(x) \overline{g(x)} d x+p_{2}^{2} \int_{\xi}^{b} f(x) \overline{g(x)} d x \\
& =0,
\end{aligned}
$$

so $f(x)=0$. For all $V=\left(\nu(x), \nu_{1}, 0\right) \in D(A),\langle F, V\rangle=$ $(\gamma / \rho) f_{1} \overline{\nu_{1}}=0, f_{1}=0$, since $\nu_{1}$ is arbitrary. Similarly, one gets $f_{2}=0$. Above all, one has $F=(0,0,0)$ which proves the assertion.

Definition 6. Let $W(f, g ; x)=f(x) g^{\prime}(x)-f^{\prime}(x) g(x)$ denote the Wronskian of functions $f(x)$ and $g(x)$. One has

$$
\begin{aligned}
& W(f, g ; a)=p_{1}^{2}[f, \bar{g}](a), \\
& W(f, g ; b)=p_{2}^{2}[f, \bar{g}](b) .
\end{aligned}
$$

Theorem 7. Operator $A$ is symmetric.

Proof. For each $F, G \in D(A)$, by the definition of inner product and operator $A$, we can get

$$
\begin{aligned}
\langle A F, G\rangle & -\langle F, A G\rangle \\
= & \gamma W(f, \bar{g} ; \xi-0)-W(f, \bar{g} ; \xi+0)+W(f, \bar{g} ; b) \\
& -\gamma W(f, \bar{g} ; a) \\
& +\frac{\gamma}{\rho}\left[T_{a}^{\prime}(f) \overline{T_{a}(g)}-T_{a}(f) \overline{T_{a}^{\prime}(g)}\right] \\
& +\frac{1}{\theta}\left[T_{b}^{\prime}(f) \overline{T_{b}(g)}-T_{b}(f) \overline{T_{b}^{\prime}(g)}\right] ;
\end{aligned}
$$

then (4) implies that

$$
W(f, \bar{g} ; \xi+0)=\gamma W(f, \bar{g} ; \xi-0) ;
$$

using boundary condition (2), we get

$$
\frac{\gamma}{\rho}\left[T_{a}^{\prime}(f) \overline{T_{a}(g)}-T_{a}(f) \overline{T_{a}^{\prime}(g)}\right]=\gamma W(f, \bar{g} ; a),
$$

and, by boundary condition (3), we have

$$
\frac{1}{\theta}\left[T_{b}^{\prime}(f) \overline{T_{b}(g)}-T_{b}(f) \overline{T_{b}^{\prime}(g)}\right]=W(f, \bar{g} ; b) .
$$

Substituting (17)-(19) into (16) yields $\langle A F, G\rangle-\langle F, A G\rangle=0$. This completes the proof.

Moreover, we have the following conclusion.

\section{Theorem 8. Operator $A$ is self-adjoint.}

Proof. $A$ is self-adjoint if and only if, for each $F=$ $\left(f(x), T_{a}(f), T_{b}(f)\right) \in D(A),\langle A F, W\rangle=\langle F, U\rangle$ for some $U \in H$ implying that $W \in D(A)$ and $A W=U$, where $W=(w(x), h, r), U=(\mu(x), k, s)$. Concretely, we should prove that the following properties hold:

(i) $w(x), w^{\prime}(x)$ are absolutely continuous on $(a, \xi) \cup$ $(\xi, b), L w \in L^{2}((a, \xi) \cup(\xi, b))$.

(ii) $h=b_{1}[w, u](a)-b_{2}[w, v](a), r=d_{1}[w, u](b)-d_{2}[w$, $v](b)$.

(iii) $L_{i} w=0, i=3,4$.

(iv) $\mu(x)=L w$.

(v) $k=a_{1}[w, u](a)-a_{2}[w, v](a) ; s=c_{1}[w, u](b)-c_{2}[w$, $v](b)$.

For an arbitrary point $F \in C_{0}^{\infty} \oplus 0 \oplus 0 \subset D(A)$, we have

$$
\begin{gathered}
\gamma p_{1}^{2} \int_{a}^{\xi}(L f(x)) \overline{w(x)} d x+p_{2}^{2} \int_{\xi}^{b}(L f(x)) \overline{w(x)} d x \\
\quad=\gamma p_{1}^{2} \int_{a}^{\xi} f(x) \overline{\mu(x)} d x+p_{2}^{2} \int_{\xi}^{b} f(x) \overline{\mu(x)} d x
\end{gathered}
$$

that is, $\langle L f, w\rangle_{1}=\langle f, \mu\rangle_{1}$. According to the classical SturmLiouville theory, (i) and (iv) hold. So $\langle A F, W\rangle=\langle F, U\rangle, \forall F \in$ $D(A)$.

$$
\begin{aligned}
& \langle L f, w\rangle_{1}+\frac{\gamma}{\rho} T_{a}^{\prime}(f) \bar{h}+\frac{1}{\theta} T_{b}^{\prime}(f) \bar{r} \\
& =\langle f, L w\rangle_{1}+\frac{\gamma}{\rho} T_{a}(f) \bar{k}+\frac{1}{\theta} T_{b}(f) \bar{s} .
\end{aligned}
$$

Besides, we have

$$
\begin{aligned}
\langle L f, w\rangle_{1}= & \langle f, L w\rangle_{1}+\gamma W(f, \bar{w} ; \xi-0) \\
& -W(f, \bar{w} ; \xi+0)+W(f, \bar{w} ; b) \\
& -\gamma W(f, \bar{w} ; a) .
\end{aligned}
$$

Substituting (22) into (21), we get

$$
\begin{aligned}
\frac{\gamma}{\rho}\left(T_{a}(f) \bar{k}-T_{a}^{\prime}(f) \bar{h}\right)+\frac{1}{\theta}\left(T_{b}(f) \bar{s}-T_{b}^{\prime}(f) \bar{r}\right) \\
=\gamma W(f, \bar{w} ; \xi-0)-W(f, \bar{w} ; \xi+0)+W(f, \bar{w} ; b) \\
\quad-\gamma W(f, \bar{w} ; a) .
\end{aligned}
$$


By Naimark's Patching Lemma [4], there exists $F \in D(A)$ such that

$$
\begin{aligned}
& {[f, u](b)=[f, v](b)=f(\xi \pm 0)=f^{\prime}(\xi \pm 0)=0,} \\
& {[f, u](a)=b_{2},} \\
& {[f, v](a)=b_{1} .}
\end{aligned}
$$

Substituting (24) into (23), we have $h=b_{1}[w, u](a)-$ $b_{2}[w, v](a)$. Further, there exists $F \in D(A)$ such that

$$
\begin{aligned}
& {[f, u](a)=[f, v](a)=f(\xi \pm 0)=f^{\prime}(\xi \pm 0)=0,} \\
& {[f, u](b)=d_{2},} \\
& {[f, v](b)=d_{1} .}
\end{aligned}
$$

Analogously, we can get $r=d_{1}[w, u](b)-d_{2}[w, v](b)$. So (ii) holds. Similarly, one proves (v). Next, let $F \in D(A)$ such that

$$
\begin{aligned}
{[f, u](a) } & =[f, v](a)=[f, u](b)=[f, v](b) \\
& =f(\xi+0)=0, \\
f(\xi-0) & =-\delta_{2}, \\
f^{\prime}(\xi-0) & =\delta_{1}, \\
f^{\prime}(\xi+0) & =\gamma .
\end{aligned}
$$

Then, by (23), we have $L_{3} w=0$. Similarly, we can get $L_{4} w=$ 0 . So $A$ is a self-adjoint operator.

From the properties of self-adjoint operators, we have the following corollaries.

Corollary 9. All eigenvalues of the singular Sturm-Liouville problem (1)-(4) are real.

Corollary 10. Let $\lambda_{1}$ and $\lambda_{2}$ be two different eigenvalues of the singular Sturm-Liouville problem (1)-(4); then the corresponding eigenfunctions $f(x)$ and $g(x)$ are orthogonal in the sense of

$$
\begin{aligned}
& \gamma p_{1}^{2} \int_{a}^{\xi} f(x) \overline{g(x)} d x+p_{2}^{2} \int_{\xi}^{b} f(x) \overline{g(x)} d x+\frac{\gamma}{\rho} f_{1} \overline{g_{1}} \\
& +\frac{1}{\theta} f_{2} \overline{g_{2}}=0 .
\end{aligned}
$$

\section{Asymptotic Approximation of Fundamental Solutions}

In this section, we construct the fundamental solutions of problem (1)-(4) and get the asymptotic approximation for fundamental solutions.

Lemma 11 (see [5]). Let the real-valued function $q(x)$ be continuous on $I=(a, \xi) \cup(\xi, b)$, and let $f(\lambda), g(\lambda)$ be given entire functions. Then, for any $\lambda \in \mathbb{C}$, the equation

$$
\begin{aligned}
L y:=-\left(p(x) y^{\prime}(x)\right)^{\prime}+q(x) y(x) & =\lambda y(x), \\
& x \in(a, \xi) \cup(\xi, b)
\end{aligned}
$$

has unique solution $y=y(x, \lambda)$ satisfying the initial conditions

$$
\begin{aligned}
y(a, \lambda) & =f(\lambda), \\
y^{\prime}(a, \lambda) & =g(\lambda) .
\end{aligned}
$$

For each fixed $x \in(a, \xi) \cup(\xi, b), y(x, \lambda)$ is an entire function of $\lambda$.

Here, we define fundamental solutions $\varphi(x, \lambda)$ and $\chi(x, \lambda)$ of (1) by the following procedure:

$$
\begin{aligned}
& \varphi(x, \lambda)= \begin{cases}\varphi_{1}(x, \lambda), & x \in(a, \xi), \\
\varphi_{2}(x, \lambda), & x \in(\xi, b),\end{cases} \\
& \chi(x, \lambda)= \begin{cases}\chi_{1}(x, \lambda), & x \in(a, \xi), \\
\chi_{2}(x, \lambda), & x \in(\xi, b) .\end{cases}
\end{aligned}
$$

Let $\varphi_{1}(x, \lambda)$ be the solution of (1) on the interval $(a, \xi)$, which satisfies the initial conditions

$$
\begin{aligned}
& \varphi_{1}(a, \lambda):=[y, u](a, \lambda)=a_{2}-\lambda b_{2} \\
& \varphi_{1}^{\prime}(a, \lambda):=[y, v](a, \lambda)=a_{1}-\lambda b_{1}
\end{aligned}
$$

by virtue of Lemma 11 , we can define the solution $\varphi_{2}(x, \lambda)$ of (1) on $(\xi, b)$ by the initial conditions

$$
\left(\begin{array}{c}
\varphi_{2}(\xi+0) \\
\varphi_{2}^{\prime}(\xi+0)
\end{array}\right)=\left(\begin{array}{l}
\delta_{1} \varphi_{1}(\xi-0)+\delta_{2} \varphi_{1}^{\prime}(\xi-0) \\
\delta_{3} \varphi_{1}(\xi-0)+\delta_{4} \varphi_{1}^{\prime}(\xi-0)
\end{array}\right)
$$

Analogously, we define the solutions $\chi_{2}(x, \lambda)$ and $\chi_{1}(x, \lambda)$ of (1) by the initial conditions

$$
\begin{aligned}
\chi_{2}(b, \lambda) & :=[y, u](b, \lambda)=c_{2}-\lambda d_{2}, \\
\chi_{2}^{\prime}(b, \lambda) & :=[y, v](b, \lambda)=c_{1}-\lambda d_{1}, \\
\left(\begin{array}{c}
\chi_{1}(\xi-0) \\
\chi_{1}^{\prime}(\xi-0)
\end{array}\right) & =\left(\begin{array}{c}
\frac{\delta_{4} \chi_{2}(\xi+0)-\delta_{2} \chi_{2}^{\prime}(\xi+0)}{\gamma} \\
\frac{\delta_{1} \chi_{2}^{\prime}(\xi+0)-\delta_{3} \chi_{2}(\xi+0)}{\gamma}
\end{array}\right) .
\end{aligned}
$$

Now, we consider Wronskian

$$
\begin{aligned}
W_{i}(\lambda) & :=W\left(\varphi_{i}, \chi_{i} ; x\right) \\
& =\varphi_{i}(x, \lambda) \chi_{i}^{\prime}(x, \lambda)-\varphi_{i}^{\prime}(x, \lambda) \chi_{i}(x, \lambda) \\
& (i=1,2) .
\end{aligned}
$$

By the dependence of solutions of initial value problems on the parameter, one has that $W_{i}(\lambda)(i=1,2)$ are entire functions of $\lambda$ and are independent of $x$.

Lemma 12. For every $\lambda \in \mathbb{C}, W_{2}(\lambda)=\gamma W_{1}(\lambda)$. 
Proof. By the definition of $W_{i}(\lambda)$, we have

$$
\begin{aligned}
W_{1}(\lambda)= & \varphi_{1}(\xi-0, \lambda) \chi_{1}^{\prime}(\xi-0, \lambda) \\
& -\varphi_{1}^{\prime}(\xi-0, \lambda) \chi_{1}(\xi-0, \lambda), \\
W_{2}(\lambda)= & \varphi_{2}(\xi+0, \lambda) \chi_{2}^{\prime}(\xi+0, \lambda) \\
& -\varphi_{2}^{\prime}(\xi+0, \lambda) \chi_{2}(\xi+0, \lambda) ;
\end{aligned}
$$

using the transmission conditions (4), simple computation gives

$$
W\left(\varphi_{2}, \chi_{2} ; \xi+0\right)=\gamma W\left(\varphi_{1}, \chi_{1} ; \xi-0\right)
$$

Thus, for each $\lambda \in \mathbb{C}$, we have $W_{2}(\lambda)=\gamma W_{1}(\lambda)$. This completes the proof.

Besides, we set $W(\lambda):=W_{1}(\lambda)=(1 / \gamma) w_{2}(\lambda)$.

Theorem 13. The eigenvalues of problem (1)-(4) coincide with the zeros of the function $W(\lambda)$.

Proof. Let $\nu_{0}\left(x, \lambda_{0}\right)$ be any eigenfunction corresponding to eigenvalue $\lambda_{0}$; then the function $\nu_{0}\left(x, \lambda_{0}\right)$ may be represented in the form

$$
\begin{aligned}
v_{0}\left(x, \lambda_{0}\right) \\
= \begin{cases}m_{1} \varphi_{1}\left(x, \lambda_{0}\right)+m_{2} \chi_{1}\left(x, \lambda_{0}\right), & x \in(a, \xi), \\
m_{3} \varphi_{2}\left(x, \lambda_{0}\right)+m_{4} \chi_{2}\left(x, \lambda_{0}\right), & x \in(\xi, b),\end{cases}
\end{aligned}
$$

where at least one of the constants $m_{i}(i=1,2,3,4)$ is not zero. We should show that $W\left(\lambda_{0}\right)=0$. Suppose to the contrary that there exists $\lambda_{0} \in R$ such that $W\left(\lambda_{0}\right)=W_{1}\left(\lambda_{0}\right)=$ $(1 / \gamma) W_{2}\left(\lambda_{0}\right) \neq 0$. Since eigenfunction $\nu_{0}\left(x, \lambda_{0}\right)$ satisfies both boundary and transmission conditions (2)-(4), we have $L_{i} \nu_{0}\left(x, \lambda_{0}\right)=0(i=1,2,3,4)$, while the determinant of coefficient matrix is not zero, so we obtain $m_{i}=0(i=$ $1,2,3,4)$, which is a contradiction; then $W\left(\lambda_{0}\right)=0$. Conversely, let $\lambda=\lambda_{0}$ be a zero of function $W(\lambda)$; then $W\left(\lambda_{0}\right)=W_{1}\left(\lambda_{0}\right)=(1 / \gamma) W_{2}\left(\lambda_{0}\right)=0$; therefore, $\chi_{i}\left(x, \lambda_{0}\right)=$ $k \varphi_{i}\left(x, \lambda_{0}\right)(i=1,2)$, for some $k \neq 0$. Since both $\varphi_{2}\left(x, \lambda_{0}\right)$ and $\chi_{2}\left(x, \lambda_{0}\right)$ satisfy the boundary condition (3),

$$
\varphi\left(x, \lambda_{0}\right)= \begin{cases}\varphi_{1}\left(x, \lambda_{0}\right), & x \in(a, \xi), \\ \varphi_{2}\left(x, \lambda_{0}\right), & x \in(\xi, b)\end{cases}
$$

satisfies problem (1)-(4). So function $\varphi\left(x, \lambda_{0}\right)$ is an eigenfunction of problem (1)-(4) corresponding to eigenvalue $\lambda_{0}$. This completes the proof.

Theorem 14. The eigenvalues of problem (1)-(4) are analytically single.

Proof. Let $\lambda=s+i t$, and we use the following notions for simplicity: $\varphi=\varphi(x, \lambda), \varphi_{1 \lambda}=\partial \varphi_{1} / \partial \lambda$, and $\varphi_{1 \lambda}^{\prime}=\partial \varphi_{1}^{\prime} / \partial \lambda$.
We differentiate the equation $A \chi=\lambda \chi$ with respect to $\lambda$ to obtain

$$
A \chi_{\lambda}=\chi+\lambda \chi_{\lambda}
$$

Using integration by parts, we get

$$
\begin{aligned}
& \left\langle A \chi_{\lambda}, \varphi\right\rangle_{1}-\left\langle\chi_{\lambda}, A \varphi\right\rangle_{1} \\
& \quad=\gamma\left(\chi_{1 \lambda} \overline{\varphi_{1}^{\prime}}-\left.\chi_{1 \lambda}^{\prime} \overline{\varphi_{1}}\right|_{a} ^{\xi}\right)+\left.\left(\chi_{2 \lambda} \overline{\varphi_{2}^{\prime}}-\chi_{2 \lambda}^{\prime} \overline{\varphi_{2}}\right)\right|_{\xi} ^{b}
\end{aligned}
$$

Substituting (39) and $A \varphi=\lambda \varphi$ into the left side of (40), we have

$$
\left\langle\lambda \chi_{\lambda}, \varphi\right\rangle_{1}-\left\langle\chi_{\lambda}, \lambda \varphi\right\rangle_{1}=\langle\chi, \varphi\rangle_{1}+2 i t\left\langle\chi_{\lambda}, \varphi\right\rangle_{1}
$$

Moreover,

$$
\begin{aligned}
\gamma\left(\chi_{1 \lambda} \overline{\varphi_{1}^{\prime}}-\left.\chi_{1 \lambda}^{\prime} \overline{\varphi_{1}}\right|_{a} ^{\xi}\right)+\left.\left(\chi_{2 \lambda} \overline{\varphi_{2}^{\prime}}-\chi_{2 \lambda}^{\prime} \overline{\varphi_{2}}\right)\right|_{\xi} ^{b} \\
=d_{1} \overline{\varphi_{2}}(b)-d_{2} \overline{\varphi_{2}^{\prime}}(b) \\
\quad-\gamma\left[\left(a_{1}-\lambda b_{1}\right) \chi_{1 \lambda}(a)-\left(a_{2}-\lambda b_{2}\right) \chi_{1 \lambda}^{\prime}(a)\right] .
\end{aligned}
$$

By (31), we observe that

$$
\begin{aligned}
W^{\prime}(\lambda)= & \varphi_{1}(a, \lambda) \chi_{1}^{\prime}(a, \lambda)-\varphi_{1}^{\prime}(a, \lambda) \chi_{1}(a, \lambda) \\
= & b_{1} \chi_{1}(a)-b_{2} \chi_{1}^{\prime}(a)+\left(a_{2}-\lambda b_{2}\right) \chi_{1 \lambda}^{\prime}(a) \\
& -\left(a_{1}-\lambda b_{1}\right) \chi_{1 \lambda}(a) ;
\end{aligned}
$$

then (40) becomes

$$
\begin{aligned}
\gamma W^{\prime}(\lambda)= & \langle\chi, \varphi\rangle_{1}+2 i t\left\langle\chi_{\lambda}, \varphi\right\rangle_{1} \\
& +\gamma\left(b_{1} \chi_{1}(a)-b_{2} \chi_{1}^{\prime}(a)\right) \\
& -\left(d_{1} \bar{\varphi}_{2}(b)-d_{2} \bar{\varphi}_{2}^{\prime}(b)\right) .
\end{aligned}
$$

Next, let $\mu_{0}$ be an arbitrary zero of $W(\lambda)$. Since $W\left(\mu_{0}\right)=0$, we obtain $\varphi_{i}\left(x, \mu_{0}\right)=k \chi_{i}\left(x, \mu_{0}\right)(i=1,2)(k \neq 0), k \in \mathbb{R}$. Noting that $\mu_{0}$ is real, a short calculation (44) becomes

$$
\begin{aligned}
\gamma W^{\prime}\left(\mu_{0}\right) & \\
= & k\left(\gamma p_{1}^{2} \int_{a}^{\xi}\left|\chi_{1}(x)\right|^{2} d x+p_{2}^{2} \int_{\xi}^{b}\left|\chi_{2}(x)\right|^{2} d x\right) \\
& +\frac{\rho}{k}+\theta k
\end{aligned}
$$

Since $\rho>0, \theta>0, \gamma>0$, and $k \neq 0, W^{\prime}\left(\mu_{0}\right) \neq 0$. Hence, the analytic multiplicity of $\mu_{0}$ is one, which completes the proof. 
Lemma 15. Let $\lambda=s^{2}, s=\sigma+i$. Then the following integral and differential equations hold for $k=0$ and $k=1$ :

$$
\begin{aligned}
& \frac{d^{k}}{d x^{k}} \varphi_{1}(x, \lambda) \\
& =\left(a_{2}-s^{2} b_{2}\right) \frac{d^{k}}{d x^{k}} \cos \left[p_{1} s(x-a)\right] \\
& \quad-\frac{a_{1}-s^{2} b_{1}}{p_{1} s} \frac{d^{k}}{d x^{k}} \sin \left[p_{1} s(x-a)\right] \\
& \quad+\frac{p_{1}}{s} \int_{a}^{x} \frac{d^{k}}{d x^{k}} \sin \left[p_{1} s(x-\tau)\right] q(\tau) \varphi_{1}(\tau) d \tau, \\
& \frac{d^{k}}{d x^{k}} \varphi_{2}(x, \lambda) \\
& =\varphi_{2}(\xi+0) \frac{d^{k}}{d x^{k}} \cos \left[p_{2} s(x-\xi)\right] \\
& \quad+\frac{1}{p_{2} s} \varphi_{2}^{\prime}(\xi+0) \frac{d^{k}}{d x^{k}} \sin \left[p_{2} s(x-\xi)\right] \\
& \quad+\frac{p_{2}}{s} \int_{\xi}^{x} \frac{d^{k}}{d x^{k}} \sin \left[p_{2} s(x-\tau)\right] q(\tau) \varphi_{2}(\tau) d \tau .
\end{aligned}
$$

Proof. For the case of $k=0$, consider $\varphi_{1}(x, \lambda)$ as the solution of the following nonhomogeneous problem:

$$
\begin{aligned}
-\left(p(x) y(x)^{\prime}\right)^{\prime}+\lambda y(x) & =q(x) y(x) \\
\varphi_{1}(a, \lambda) & =a_{2}-\lambda b_{2}, \\
\varphi_{1}^{\prime}(a, \lambda) & =a_{1}-\lambda b_{1} .
\end{aligned}
$$

Using the method of constant variation, $\varphi_{1}(x, \lambda)$ satisfies

$$
\begin{aligned}
\varphi_{1}(x, \lambda)= & \left(a_{2}-s^{2} b_{2}\right) \cos \left[p_{1} s(x-a)\right] \\
& -\frac{a_{1}-s^{2} b_{1}}{p_{1} s} \frac{d^{k}}{d x^{k}} \sin \left[p_{1} s(x-a)\right] \\
& +\frac{p_{1}}{s} \int_{a}^{x} \sin \left[p_{1} s(x-\tau)\right] q(\tau) \varphi_{1}(\tau) d \tau .
\end{aligned}
$$

Then, differentiating it with respect to $x$, we have (46). The proof for (47) is similar, so we omit the details.

Similarly, we have the following theorem.

Lemma 16. Let $\lambda=s^{2}, s=\sigma+i$. Then the following integral and differential equations hold for $k=0$ and $k=1$ :

$$
\begin{aligned}
& \frac{d^{k}}{d x^{k}} \chi_{1}(x, \lambda)=\frac{\delta_{4} \chi_{2}(\xi+0)-\delta_{2} \chi_{2}^{\prime}(\xi+0)}{\gamma} \frac{d^{k}}{d x^{k}} \\
& \cdot \cos \left[p_{1} s(x-\xi)\right]+\frac{\delta_{1} \chi_{2}^{\prime}(\xi+0)-\delta_{3} \chi_{2}(\xi+0)}{p_{1} s \gamma}
\end{aligned}
$$

$$
\begin{gathered}
\cdot \frac{d^{k}}{d x^{k}} \sin \left[p_{1} s(x-\xi)\right]-\frac{p_{1}}{s} \\
\cdot \int_{x}^{\xi} \frac{d^{k}}{d x^{k}} \sin \left[p_{1} s(x-\tau)\right] q(\tau) \chi_{1}(\tau) d \tau, \\
\frac{d^{k}}{d x^{k}} \chi_{2}(x, \lambda)=\left(c_{2}-s^{2} d_{2}\right) \frac{d^{k}}{d x^{k}} \cos \left[p_{2} s(x-b)\right] \\
+\frac{c_{1}-s^{2} d_{1}}{p_{2} s} \frac{d^{k}}{d x^{k}} \sin \left[p_{2} s(x-b)\right]-\frac{p_{2}}{s} \\
\cdot \int_{x}^{b} \frac{d^{k}}{d x^{k}} \sin \left[p_{2} s(x-\tau)\right] q(\tau) \chi_{2}(\tau) d \tau .
\end{gathered}
$$

Lemma 17. Let $\lambda=s^{2}, s=\sigma+i t$. Then, for $k=0,1, \varphi(x, \lambda)$ have the following estimations.

Case 1. If $b_{2} \neq 0$, then

$$
\begin{aligned}
& \frac{d^{k}}{d x^{k}} \varphi_{1}(x, \lambda) \\
& =-s^{2} b_{2} \frac{d^{k}}{d x^{k}} \cos \left[p_{1} s(x-a)\right] \\
& \quad+O\left(|s|^{k+1} e^{|t| p_{1}(x-a)}\right), \\
& \frac{d^{k}}{d x^{k}} \varphi_{2}(x, \lambda) \\
& =p_{1} \delta_{2} s^{3} b_{2} \sin \left[p_{1} s(\xi-a)\right] \frac{d^{k}}{d x^{k}} \cos \left[p_{2} s(x-a)\right] \\
& \quad+O\left(|s|^{k+2} e^{|t|\left(p_{1}(\xi-a)+p_{2}(x-a)\right)}\right) .
\end{aligned}
$$

Case 2. If $b_{2}=0$, then

$$
\begin{aligned}
& \frac{d^{k}}{d x^{k}} \varphi_{1}(x, \lambda) \\
& =-\frac{b_{1} s}{p_{1}} \frac{d^{k}}{d x^{k}} \sin \left[p_{1} s(x-a)\right]+O\left(|s|^{k} e^{|t| p_{1}(x-a)}\right), \\
& \frac{d^{k}}{d x^{k}} \varphi_{2}(x, \lambda) \\
& =-\delta_{2} b_{1} s^{2} \cos \left[p_{1} s(\xi-a)\right] \frac{d^{k}}{d x^{k}} \cos \left[p_{2} s(x-\xi)\right] \\
& \quad+O\left(|s|^{k+1} e^{|t|\left(p_{1}(\xi-a)+p_{2}(x-\xi)\right)}\right) .
\end{aligned}
$$

Each of these asymptotic equalities holds uniformly for $x$ as $|\lambda| \rightarrow \infty$.

Proof. The proof of formulas for $\varphi_{1}(x, \lambda)$ is identical to those of Titchmarsh's proof for $\varphi(x, \lambda)$ (see [6]), so we only give the proof of formulas for $\varphi_{2}(x, \lambda)$, namely, equality (51); the other equalities are similar. 

have

For $k=0$, by the estimations of $\varphi_{1}(x, \lambda)$ and $\varphi_{1}^{\prime}(x, \lambda)$, we

$$
\begin{aligned}
\varphi_{1}(\xi-0, \lambda)= & -s^{2} b_{2} \cos \left[p_{1} s(\xi-a)\right] \\
& +O\left(|s| e^{|t| p_{1}(\xi-a)}\right), \\
\varphi_{1}^{\prime}(\xi-0, \lambda)= & p_{1} s^{3} b_{2} \sin \left[p_{1} s(\xi-a)\right] \\
& +O\left(|s|^{2} e^{|t| p_{1}(\xi-a)}\right) ;
\end{aligned}
$$

then, substituting (53) into (47) and observing (32), we have

$$
\begin{aligned}
\varphi_{2}(x, \lambda) & \\
= & p_{1} \delta_{2} s^{3} b_{2} \sin \left[p_{1} s(\xi-a)\right] \cos \left[p_{2} s(x-a)\right] \\
& +O\left(|s|^{2} e^{|t|\left(p_{1}(\xi-a)+p_{2}(x-\xi)\right)}\right) ;
\end{aligned}
$$

differentiating (54) with respect to $x$, we have (51).

Lemma 18. Let $\lambda=s^{2}, s=\sigma+$ it. Then, for $k=0,1, \chi(x, \lambda)$ have the following estimations.

Case 1. If $d_{2} \neq 0$, then

$$
\begin{aligned}
& \frac{d^{k}}{d x^{k}} \chi_{1}(x, \lambda) \\
& =-\frac{p_{2} d_{2} \delta_{2} s^{3}}{\gamma} \sin \left[p_{2} s(\xi-b)\right] \frac{d^{k}}{d x^{k}} \cos \left[p_{1} s(x-\xi)\right] \\
& \quad+O\left(|s|^{k+2} e^{|t|\left(p_{1}(x-\xi)+p_{2}(\xi-b)\right)}\right), \\
& \frac{d^{k}}{d x^{k}} \chi_{2}(x, \lambda) \\
& =-d_{2} s^{2} \frac{d^{k}}{d x^{k}} \cos \left[p_{2} s(x-b)\right] \\
& \quad+O\left(|s|^{k+1} e^{|t| p_{2}(x-b)}\right) .
\end{aligned}
$$

Case 2. If $d_{2}=0$, then

$$
\begin{aligned}
& \frac{d^{k}}{d x^{k}} \chi_{1}(x, \lambda) \\
& =-\frac{d_{1} \delta_{2} s^{2}}{\gamma} \cos \left[p_{2} s(\xi-b)\right] \frac{d^{k}}{d x^{k}} \cos \left[p_{1} s(x-\xi)\right] \\
& \quad+O\left(|s|^{k+1} e^{|t|\left(p_{1}(x-\xi)+p_{2}(\xi-b)\right)}\right), \\
& \frac{d^{k}}{d x^{k}} \chi_{2}(x, \lambda) \\
& =-\frac{d_{1} s}{p_{2}} \frac{d^{k}}{d x^{k}} \sin \left[p_{2} s(x-b)\right]+O\left(|s|^{k} e^{|t| p_{2}(x-b)}\right) .
\end{aligned}
$$

Each of these asymptotic equalities holds uniformly for $x$ as $|\lambda| \rightarrow \infty$.

Theorem 19. Let $\lambda=s^{2}, s=\sigma+i$. Then the function $W_{2}(\lambda)$ has the following asymptotic representations.
Case 1. If $b_{2} \neq 0$ and $d_{2} \neq 0$, then

$$
\begin{aligned}
& W_{2}(\lambda) \\
& =\delta_{2} p_{1} p_{2} b_{2} d_{2} s^{6} \sin \left[p_{1} s(\xi-a)\right] \sin \left[p_{2} s(b-\xi)\right] \\
& \quad+O\left(|s|^{5} e^{|t|\left(p_{1}(\xi-a)+p_{2}(b-\xi)\right)}\right) .
\end{aligned}
$$

Case 2. If $b_{2} \neq 0$ and $d_{2}=0$, then

$W_{2}(\lambda)$

$$
\begin{aligned}
= & -\delta_{2} p_{1} b_{2} d_{1} s^{5} \sin \left[p_{1} s(\xi-a)\right] \cos \left[p_{2} s(b-\xi)\right] \\
& +O\left(|s|^{4} e^{|t|\left(p_{1}(\xi-a)+p_{2}(b-\xi)\right)}\right) .
\end{aligned}
$$

Case 3. If $b_{2}=0$ and $d_{2} \neq 0$, then

$$
\begin{aligned}
W_{2}(\lambda) & \\
= & -\delta_{2} p_{2} b_{1} d_{2} s^{5} \cos \left[p_{1} s(\xi-a)\right] \sin \left[p_{2} s(b-\xi)\right] \\
& +O\left(|s|^{4} e^{|t|\left(p_{1}(\xi-a)+p_{2}(b-\xi)\right)}\right) .
\end{aligned}
$$

Case 4. If $b_{2}=0$ and $d_{2}=0$, then

$$
\begin{aligned}
W_{2}(\lambda)= & \delta_{2} b_{1} d_{1} s^{4} \cos \left[p_{1} s(\xi-a)\right] \cos \left[p_{2} s(b-\xi)\right] \\
& +O\left(|s|^{3} e^{|t|\left(p_{1}(\xi-a)+p_{2}(b-\xi)\right)}\right) .
\end{aligned}
$$

Proof. By the definition of $W_{2}(\lambda)$, we have

$$
\begin{aligned}
W_{2}(\lambda) & =\varphi_{2}(b, \lambda) \chi_{2}^{\prime}(b, \lambda)-\varphi_{2}^{\prime}(b, \lambda) \chi_{2}(b, \lambda) \\
& =\left(c_{1}-\lambda d_{1}\right) \varphi_{2}(b, \lambda)-\left(c_{2}-\lambda d_{2}\right) \varphi_{2}^{\prime}(b, \lambda) .
\end{aligned}
$$

According to the equalities of $\varphi_{2}(x, \lambda)$ and $\varphi_{2}^{\prime}(x, \lambda)$ in Lemma 17, we can obtain the formulas of $W_{2}(\lambda)$ in this theorem.

Corollary 20. The eigenvalues of the boundary value problem (1)-(4) are bounded below.

Proof. Putting $s=$ it $(t>0)$ in Theorem 19, we can obtain $W_{2}(\lambda)=W_{2}\left(-t^{2}\right) \rightarrow \infty(t \rightarrow \infty)$. Hence, $W_{2}\left(-t^{2}\right) \neq 0$ for sufficiently negative $\lambda$ and sufficiently large $\lambda$. This completes the proof.

\section{Asymptotic Formulas for Eigenvalues and Eigenfunctions}

In this section, we can get the asymptotic formulas for the eigenvalues and eigenfunctions of the singular SturmLiouville problem (1)-(4). Since the eigenvalues coincide with the zeros of the entire function $W(\lambda)$, it follows that they have no finite limits.

Theorem 21. The eigenvalues $\lambda_{n}=s_{n}^{2}(n=0,1,2, \ldots)$ of problem (1)-(4) have the following asymptotic representations as $n \rightarrow \infty$. 
Case 1. If $b_{2} \neq 0$ and $d_{2} \neq 0$, then

$$
\begin{aligned}
& s_{n}^{\prime}=\frac{(n-1) \pi}{p_{1}(\xi-a)}+O\left(\frac{1}{n}\right), \\
& s_{n}^{\prime \prime}=\frac{(n-1) \pi}{p_{2}(b-\xi)}+O\left(\frac{1}{n}\right) .
\end{aligned}
$$

Case 2. If $b_{2} \neq 0$ and $d_{2}=0$, then

$$
\begin{aligned}
& s_{n}^{\prime}=\frac{(n-1) \pi}{p_{1}(\xi-a)}+O\left(\frac{1}{n}\right), \\
& s_{n}^{\prime \prime}=\frac{(n-1 / 2) \pi}{p_{2}(b-\xi)}+O\left(\frac{1}{n}\right) .
\end{aligned}
$$

Case 3. If $b_{2}=0$ and $d_{2} \neq 0$, then

$$
\begin{aligned}
& s_{n}^{\prime}=\frac{(n-1 / 2) \pi}{p_{1}(\xi-a)}+O\left(\frac{1}{n}\right), \\
& s_{n}^{\prime \prime}=\frac{(n-1) \pi}{p_{2}(b-\xi)}+O\left(\frac{1}{n}\right) .
\end{aligned}
$$

Case 4. If $b_{2}=0$ and $d_{2}=0$, then

$$
\begin{aligned}
& s_{n}^{\prime}=\frac{(n-1 / 2) \pi}{p_{1}(\xi-a)}+O\left(\frac{1}{n}\right), \\
& s_{n}^{\prime \prime}=\frac{(n-1 / 2) \pi}{p_{2}(b-\xi)}+O\left(\frac{1}{n}\right) .
\end{aligned}
$$

Proof. By applying the well-known Rouche theorem, we can obtain these conclusions (see [3] Theorem 2.3).

According to Theorem 21 and Lemmas 17 and 18, we can obtain the following asymptotic representations of the eigenfunctions $\varphi\left(x, \lambda_{n}\right)$ and $\chi\left(x, \lambda_{n}\right)$.

Theorem 22. The eigenfunctions $\varphi\left(x, \lambda_{n}\right)$ and $\chi\left(x, \lambda_{n}\right)(n=$ $0,1,2, \ldots)$ of problem (1)-(4) have the following asymptotic representations as $n \rightarrow \infty$.

Case 1. If $b_{2} \neq 0$ and $d_{2} \neq 0$, then

$$
\begin{aligned}
& \varphi\left(x, \lambda_{n}^{\prime}\right)= \begin{cases}-b_{2}\left[\frac{(n-1) \pi}{p_{1}(\xi-a)}\right]^{2} \cos \left[\frac{(n-1) \pi}{\xi-a}(x-a)\right]+O(n), & x \in(a, \xi), \\
O\left(n^{2}\right), & x \in(\xi, b),\end{cases} \\
& \varphi\left(x, \lambda_{n}^{\prime \prime}\right)= \begin{cases}-b_{2}\left[\frac{(n-1) \pi}{p_{2}(b-\xi)}\right]^{2} \cos \left[\frac{p_{1}(n-1) \pi}{p_{2}(b-\xi)}(x-a)\right]+O(n), & x \in(a, \xi), \\
p_{1} b_{2} \delta_{2}\left[\frac{(n-1) \pi}{p_{2}(b-\xi)}\right]^{3} \sin \left[\frac{p_{1}(n-1) \pi}{p_{2}(b-\xi)}(\xi-a)\right] \cos \left[\frac{(n-1) \pi}{b-\xi}(x-a)\right]+O\left(n^{2}\right), & x \in(\xi, b),\end{cases} \\
& \chi\left(x, \lambda_{n}^{\prime}\right)= \begin{cases}-\frac{p_{2} d_{2} \delta_{2}}{\gamma}\left[\frac{(n-1) \pi}{p_{1}(\xi-a)}\right]^{3} \sin \left[\frac{p_{2}(n-1) \pi}{p_{1}(\xi-a)}\right] \cos \left[\frac{(n-1) \pi}{\xi-a}(x-\xi)\right]+O\left(n^{2}\right), & x \in(a, \xi), \\
-d_{2}\left[\frac{(n-1) \pi}{p_{1}(\xi-a)}\right]^{2} \cos \left[\frac{p_{2}(n-1) \pi}{p_{1}(\xi-a)}(x-b)\right]+O(n), & x \in(\xi, b),\end{cases} \\
& \chi\left(x, \lambda_{n}^{\prime \prime}\right)= \begin{cases}O\left(n^{2}\right), & x \in(a, \xi), \\
-d_{2}\left[\frac{(n-1) \pi}{p_{2}(b-\xi)}\right]^{2} \cos \left[\frac{(n-1) \pi}{b-\xi}(x-b)\right]+O(n), & x \in(\xi, b) .\end{cases}
\end{aligned}
$$

Case 2. If $b_{2} \neq 0$ and $d_{2}=0$, then

$$
\varphi\left(x, \lambda_{n}^{\prime}\right)= \begin{cases}-b_{2}\left[\frac{(n-1) \pi}{p_{1}(\xi-a)}\right]^{2} \cos \left[\frac{(n-1) \pi}{\xi-a}(x-a)\right]+O(n), & x \in(a, \xi), \\ O\left(n^{2}\right), & x \in(\xi, b),\end{cases}
$$




$$
\begin{aligned}
& \varphi\left(x, \lambda_{n}^{\prime \prime}\right)= \begin{cases}-b_{2}\left[\frac{(n-1 / 2) \pi}{p_{2}(b-\xi)}\right]^{2} \cos \left[\frac{p_{1}(n-1 / 2) \pi}{p_{2}(b-\xi)}(x-a)\right]+O(n), & x \in(a, \xi), \\
p_{1} b_{2} \delta_{2}\left[\frac{(n-1 / 2) \pi}{p_{2}(b-\xi)}\right]^{3} \sin \left[\frac{p_{1}(n-1 / 2) \pi}{p_{2}(b-\xi)}(\xi-a)\right] \cos \left[\frac{(n-1 / 2) \pi}{b-\xi}(x-a)\right]+O\left(n^{2}\right), & x \in(\xi, b),\end{cases} \\
& \chi\left(x, \lambda_{n}^{\prime}\right)= \begin{cases}\frac{d_{1} \delta_{2}}{\gamma}\left[\frac{(n-1) \pi}{p_{1}(\xi-a)}\right]^{2} \cos \left[\frac{p_{2}(n-1) \pi}{p_{1}(\xi-a)}\right] \cos \left[\frac{(n-1) \pi}{\xi-a}(x-\xi)\right]+O(n), & x \in(a, \xi), \\
-\frac{d_{1}}{p_{2}}\left[\frac{(n-1) \pi}{p_{1}(\xi-a)}\right] \sin \left[\frac{p_{2}(n-1) \pi}{p_{1}(\xi-a)}(x-b)\right]+O(1), & x \in(\xi, b),\end{cases} \\
& \chi\left(x, \lambda_{n}^{\prime \prime}\right)= \begin{cases}O(n), & x \in(a, \xi), \\
-\frac{d_{1}}{p_{2}}\left[\frac{(n-1 / 2) \pi}{p_{2}(b-\xi)}\right] \sin \left[\frac{(n-1 / 2) \pi}{b-\xi}(x-b)\right]+O(1), & x \in(\xi, b) .\end{cases}
\end{aligned}
$$

Case 3. If $b_{2}=0$ and $d_{2} \neq 0$, then

$$
\begin{aligned}
& \varphi\left(x, \lambda_{n}^{\prime}\right)= \begin{cases}-b_{1}\left[\frac{(n-1 / 2) \pi}{p_{1}(\xi-a)}\right] \sin \left[\frac{(n-1 / 2) \pi}{\xi-a}(x-a)\right]+O(1), & x \in(a, \xi), \\
O(n), & x \in(\xi, b),\end{cases} \\
& \varphi\left(x, \lambda_{n}^{\prime \prime}\right)= \begin{cases}-b_{1}\left[\frac{(n-1) \pi}{p_{2}(b-\xi)}\right] \sin \left[\frac{p_{1}(n-1) \pi}{p_{2}(b-\xi)}(x-a)\right]+O(1), & x \in(a, \xi), \\
-b_{1} \delta_{2}\left[\frac{(n-1) \pi}{p_{2}(b-\xi)}\right]^{2} \cos \left[\frac{p_{1}(n-1) \pi}{p_{2}(b-\xi)}(\xi-a)\right] \cos \left[\frac{(n-1) \pi}{b-\xi}(x-\xi)\right]+O(n), & x \in(\xi, b),\end{cases} \\
& \chi\left(x, \lambda_{n}^{\prime}\right)= \begin{cases}-\frac{p_{2} d_{2} \delta_{2}}{\gamma}\left[\frac{(n-1 / 2) \pi}{p_{1}(\xi-a)}\right]^{3} \sin \left[\frac{p_{2}(n-1 / 2) \pi}{p_{1}(\xi-a)}\right] \cos \left[\frac{(n-1 / 2) \pi}{\xi-a}(x-\xi)\right]+O\left(n^{2}\right), & x \in(a, \xi), \\
-d_{2}\left[\frac{(n-1 / 2) \pi}{p_{1}(\xi-a)}\right]^{2} \cos \left[\frac{p_{2}(n-1 / 2) \pi}{p_{1}(\xi-a)}(x-b)\right]+O(n), & x \in(\xi, b),\end{cases} \\
& \chi\left(x, \lambda_{n}^{\prime \prime}\right)= \begin{cases}O\left(n^{2}\right), & x \in(a, \xi), \\
-d_{2}\left[\frac{(n-1) \pi}{p_{2}(b-\xi)}\right]^{2} \cos \left[\frac{(n-1) \pi}{b-\xi}(x-b)\right]+O(n), & x \in(\xi, b) .\end{cases}
\end{aligned}
$$

Case 4. If $b_{2}=0$ and $d_{2}=0$, then

$$
\begin{aligned}
& \varphi\left(x, \lambda_{n}^{\prime}\right)= \begin{cases}-b_{1}\left[\frac{(n-1 / 2) \pi}{p_{1}(\xi-a)}\right] \sin \left[\frac{(n-1 / 2) \pi}{\xi-a}(x-a)\right]+O(1), & x \in(a, \xi), \\
O(n), & x \in(\xi, b),\end{cases} \\
& \varphi\left(x, \lambda_{n}^{\prime \prime}\right)= \begin{cases}-b_{1}\left[\frac{(n-1 / 2) \pi}{p_{2}(b-\xi)}\right] \sin \left[\frac{p_{1}(n-1 / 2) \pi}{p_{2}(b-\xi)}(x-a)\right]+O(1), & x \in(a, \xi), \\
-b_{1} \delta_{2}\left[\frac{(n-1 / 2) \pi}{p_{2}(b-\xi)}\right]^{2} \cos \left[\frac{p_{1}(n-1 / 2) \pi}{p_{2}(b-\xi)}(\xi-a)\right] \cos \left[\frac{(n-1 / 2) \pi}{b-\xi}(x-\xi)\right]+O(n), & x \in(\xi, b),\end{cases}
\end{aligned}
$$




$$
\begin{aligned}
& \chi\left(x, \lambda_{n}^{\prime}\right)= \begin{cases}\frac{d_{1} \delta_{2}}{\gamma}\left[\frac{(n-1 / 2) \pi}{p_{1}(\xi-a)}\right]^{2} \cos \left[\frac{p_{2}(n-1 / 2) \pi}{p_{1}(\xi-a)}\right] \cos \left[\frac{(n-1 / 2) \pi}{\xi-a}(x-\xi)\right]+O(n), & x \in(a, \xi), \\
-\frac{d_{1}}{p_{2}}\left[\frac{(n-1 / 2) \pi}{p_{1}(\xi-a)}\right] \sin \left[\frac{p_{2}(n-1 / 2) \pi}{p_{1}(\xi-a)}(x-b)\right]+O(1), & x \in(\xi, b),\end{cases} \\
& \chi\left(x, \lambda_{n}^{\prime \prime}\right)= \begin{cases}O(n), & x \in(a, \xi), \\
-\frac{d_{1}}{p_{2}}\left[\frac{(n-1 / 2) \pi}{p_{2}(b-\xi)}\right] \sin \left[\frac{(n-1 / 2) \pi}{b-\xi}(x-b)\right]+O(1), & x \in(\xi, b) .\end{cases}
\end{aligned}
$$

\section{Completeness of Eigenfunctions}

In this section, we get the property of spectrum for the operator $A$ and discuss the completeness of the eigenfunctions of problem (1)-(4).

Theorem 23. The operator $A$ has only point spectrum; that is, $\sigma(A)=\sigma_{p}(A)$.

Proof. We only need to prove that if $\gamma$ is not an eigenvalue of $A$, then $\gamma \in \rho(A)$. Here we investigate the equation $(A-$ $\gamma) Z=F \in H$, where $\gamma \in \mathbb{R}, Z=\left(z(x), T_{a}(z), T_{b}(z)\right)$, and $F=\left(L f, T_{a}(f), T_{b}(f)\right)$. Consider the initial value problem

$$
\begin{aligned}
-\left(p(x) z^{\prime}(x)\right)^{\prime}+q(x) z(x)-\gamma z(x) & =f(x), \\
x & \in(a, \xi) \cup(\xi, b) \\
z(\xi+0)-\delta_{1} z(\xi-0)-\delta_{2} z^{\prime}(\xi-0) & =0, \\
z^{\prime}(\xi+0)-\delta_{3} z(\xi-0)-\delta_{4} z^{\prime}(\xi-0) & =0 .
\end{aligned}
$$

Let

$$
\psi(x)= \begin{cases}\psi_{1}(x), & x \in(a, \xi), \\ \psi_{2}(x), & x \in(\xi, b)\end{cases}
$$

be the solution of the equation $-\left(p(x) z^{\prime}(x)\right)^{\prime}+q(x) z(x)-$ $\gamma z(x)=0$ satisfying the transmission conditions (4). Let

$$
\phi(x)= \begin{cases}\phi_{1}(x), & x \in(a, \xi), \\ \phi_{2}(x), & x \in(\xi, b)\end{cases}
$$

be the solution of the equation $-\left(p(x) z^{\prime}(x)\right)^{\prime}+q(x) z(x)-$ $\gamma z(x)=f(x)$ satisfying

$$
\begin{array}{r}
\phi(\xi+0)-\delta_{1} \phi(\xi-0)-\delta_{2} \phi^{\prime}(\xi-0)=0, \\
\phi^{\prime}(\xi+0)-\delta_{3} \phi(\xi-0)-\delta_{4} \phi^{\prime}(\xi-0)=0 .
\end{array}
$$

Then (70) has the general solution

$$
z(x)= \begin{cases}n \psi_{1}(x)+\phi_{1}(x), & x \in(a, \xi), \\ n \psi_{2}(x)+\phi_{2}(x), & x \in(\xi, b),\end{cases}
$$

where $n \in \mathbb{C}$.
As $\gamma$ is not an eigenvalue of problem (1)-(4), we have

$$
\begin{aligned}
\gamma & \left(b_{1}[z, u](a)-b_{2}[z, v](a)\right) \\
& -\left(a_{1}[z, u](a)-a_{2}[z, v](a)\right) \neq 0, \\
\gamma & \left(d_{1}[z, u](b)-d_{2}[z, v](b)\right) \\
& -\left(c_{1}[z, c](b)-c_{2}[z, v](b)\right) \neq 0 .
\end{aligned}
$$

The second and third components of $(A-\gamma) Z=F \in H$ mean that

$$
\begin{aligned}
& \left(a_{1}[z, u](a)-a_{2}[z, v](a)\right) \\
& \quad-\gamma\left(b_{1}[z, u](a)-b_{2}[z, v](a)\right)=T_{a}(f), \\
& \left(c_{1}[z, u](b)-c_{2}[z, v](b)\right) \\
& \quad-\gamma\left(d_{1}[z, u](b)-d_{2}[z, v](b)\right)=T_{b}(f) .
\end{aligned}
$$

Substituting (74) into (76), we can get that $n$ is uniquely solvable. So $z(x)$ is uniquely determined. Observing that $(A-$ $\gamma I)^{-1}$ is defined on all of $H$, we get that $(A-\gamma I)^{-1}$ is bounded by Theorem 8 and the closed graph theorem. Thus, $\gamma \in \rho(A)$. Hence, $\sigma(A)=\sigma_{p}(A)$.

Lemma 24. The eigenvalues of problem (1)-(4) are countably infinite and can cluster only at $\infty$.

Lemma 25. The operator $A$ has compact resolvent; that is, for each $\delta \in \mathbb{R} / \sigma_{p}(A),(A-\delta I)^{-1}$ is compact on $H$ (see [14] Theorem 6.3.3).

By the above lemmas and the spectral theorem for compact operator, we obtain the following theorem.

Theorem 26. The eigenfunctions of problem (1)-(4), expanded to become eigenfunctions of $A$, are complete in $H$; that is, let $\left\{\Phi_{n}=\left(\varphi_{n}(x), T_{a}\left(\varphi_{n}\right), T_{b}\left(\varphi_{n}\right)\right) ; n \in \mathbb{N}\right\}$ be a maximum set of orthogonal eigenfunctions of $A$, where $\left\{\varphi_{n}(x) ; n \in \mathbb{N}\right\}$ are eigenfunctions of problem (1)-(4). Then, for all $F \in H, F=$ $\sum_{n=1}^{\infty}\left\langle F, \Phi_{n}\right\rangle \Phi_{n}$.

Remark 27. In this paper, the spectral properties of singular Sturm-Liouville problems with one inner discontinuous point are considered; if there are two or even multi-inner discontinuous points $\xi_{1}, \xi_{2}, \ldots, \xi_{n}$ in the interval $(a, b)$, we 
can obtain similar results by defining a more complicated Hilbert space.

\section{Conflicts of Interest}

The authors declare that there are no conflicts of interest.

\section{Authors' Contributions}

Jinming Cai prepared the manuscript and corrected the main results and Zhaowen Zheng gave the main thought and revised the manuscript.

\section{Acknowledgments}

This research was partially supported by the NSF of China (Grants 11271225 and 11671227).

\section{References}

[1] H. Weyl, "Über gewöhnliche Differentialgleichungen mit Singularitäten und die zugehörigen Entwicklungen," Mathematische Annalen, vol. 68, no. 2, pp. 220-269, 1910.

[2] J. Weidmann, Spectral Theory of Ordinary Differential Operators, vol. 1258 of Lecture Notes in Mathematics, Springer, new York, NY, USA, 1987.

[3] Z. Cao, Ordinary Differential Operator, Shanhai Science and Technology Press, Shanghai, China, 1986 (Chinese).

[4] M. A. Naimark, Linear Differential Operators, Part 2, Harrap, London, UK, 1968.

[5] A. Zettl, Sturm-Liouville Theory, vol. 121 of Mathematical Surveys and Monographs, American Mathematics Society, Providence, RI, USA, 2005.

[6] E. C. Titchmarsh, Eigenfunction Expansions Associated with Second-Order Differential Equations, Part 1, Oxford University Press, London, UK, 1962.

[7] Z. Zheng and W. Zhang, "Characterization of eigenvalues in spectral gap for singular differential operators," Abstract and Applied Analysis, vol. 2012, Article ID 271657, 10 pages, 2012.

[8] J. Qi, Z. Zheng, and H. Sun, "Classification of Sturm-Liouville differential equations with complex coefficients and operator realizations," Proceedings of The Royal Society of London. Series A. Mathematical, Physical and Engineering Sciences, vol. 467, no. 2131, pp. 1835-1850, 2011.

[9] Z. Zheng, J. Qi, and S. Chen, "Eigenvalues below the lower bound of minimal operators of singular Hamiltonian expressions," Computers \& Mathematics with Applications, vol. 56, no. 11, pp. 2825-2833, 2008.

[10] Z. Zheng, "Invariance of deficiency indices under perturbation for discrete Hamiltonian systems," Journal of Difference Equations and Applications, vol. 19, no. 8, pp. 1243-1250, 2013.

[11] M. Demirci, Z. Akdogan, and O. S. H. Mukhtarov, "Asymptotic behavior of eigenvalues and eigenfunctions of one discontinuous boundary value problem," International Journal of Computational Cognition, vol. 2, article 3, pp. 101-113, 2004.

[12] D. Buschmann, G. Stolz, and J. Weidmann, "One-dimensional schrodinger operators with local point interactions," Journal für die Reine und Angewandte Mathematik, vol. 467, pp. 169-186, 1995.
[13] M. Kadakal, O. S. Mukhtarov, and F. S. Muhtarov, "Some spectral properties of Sturm-Liouville problem with transmission conditions," Iranian Journal of Science and Technology. Transaction A. Science, vol. 29, no. 2, pp. 229-245, 2005.

[14] A. P. Wang, Research on weimann conjecture and differential operators with transmission conditions [Ph.D. thesis], Inner Mongolia University, 2006 (Chinese).

[15] O. S. H. Mukhtarov and M. Kadakal, "Some spectral properties of one Sturm-Liouville type problem with discontinuous weight," Siberian Mathematical Journal, vol. 46, no. 4, pp. 681694, 2005.

[16] C. T. Fulton, "Two-point boundary value problems with eigenvalue parameter contained in the boundary conditions," Proceedings of the Royal Society of Edinburgh. Section A. Mathematics, vol. 77, no. 3-4, pp. 293-308, 1977.

[17] E. Şen and A. Bayramov, "Asymptotic formulations of the eigenvalues and eigenfunctions for a boundary value problem," Mathematical Methods in the Applied Sciences, vol. 36, no. 12, pp. 1512-1519, 2013.

[18] Q. Yang and W. Wang, "Asymptotic behavior of a differential operator with discontinuities at two points," Mathematical Methods in the Applied Sciences, vol. 34, no. 4, pp. 373-383, 2011.

[19] O. S. Mukhtarov, M. Kadakal, and F. S. Muhtarov, "Eigenvalues and normalized eigenfunctions of discontinuous Sturm-Liouville problem with transmission conditions," Reports on Mathematical Physics, vol. 54, no. 1, pp. 41-56, 2004.

[20] E. Şen, "A Sturm-Liouville problem with a discontinuous coefficient and containing an eigenparameter in the boundary condition," Physics Research International, vol. 2013, Article ID 159243, 9 pages, 2013.

[21] M. Kadakal and O. S. Mukhtarov, "Sturm-Liouville problems with discontinuities at two points," Computers \& Mathematics with Applications, vol. 54, no. 11-12, pp. 1367-1379, 2007.

[22] M. Kadakal and O. S. Mukhtarov, "Discontinuous Sturm-Liouville problems containing eigenparameter in the boundary conditions," Acta Mathematica Sinica, vol. 22, no. 5, pp. 1519-1528, 2006.

[23] Z. Akdoğan, M. Demirci, and O. S. H. Mukhtarov, "SturmLiouville problems with eigendependent boundary and transmissions conditions," Acta Mathematica Scientia, vol. 25, no. 4, pp. 731-740, 2005.

[24] Q. Yang and W. Wang, "Spectral properties of Sturm-Liouville operators with discontinuities at finite points," Mathematical Sciences, vol. 6, no. 1, pp. 1-9, 2012.

[25] K. Li and Z. Zheng, "Spectral properties for Sturm-Liouville equations with transmission conditions," Acta Mathematica Scientia, vol. 35, no. 5, pp. 910-926, 2015 (Chinese).

[26] F. Meng and Y. Huang, "Interval oscillation criteria for a forced second-order nonlinear differential equations with damping," Applied Mathematics and Computation, vol. 218, no. 5, pp. 18571861, 2011.

[27] Z. Zheng, X. Wang, and H. Han, "Oscillation criteria for forced second order differential equations with mixed nonlinearities," Applied Mathematics Letters, vol. 22, no. 7, pp. 1096-1101, 2009.

[28] J. Shao, F. Meng, and X. Pang, "Generalized variational oscillation principles for second-order differential equations with mixed-nonlinearities," Discrete Dynamics in Nature and Society, vol. 2012, Article ID 539213, 10 pages, 2012.

[29] J. Shao and F. Meng, "Generalized variational principles on oscillation for nonlinear nonhomogeneous differential 
equations," Abstract and Applied Analysis, vol. 2011, Article ID 972656, 10 pages, 2011.

[30] A. V. Likov and Yu. A. Mikhailov, The Theory of Heat and Mass Transfer, Qosenerqoizdat, 1963 (Russian). 


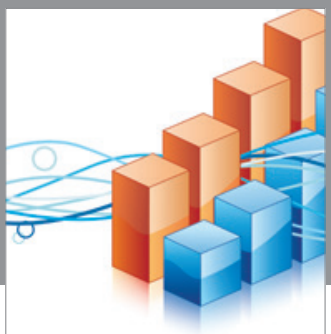

Advances in

Operations Research

vatem alat4

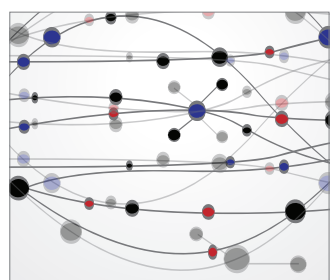

\section{The Scientific} World Journal
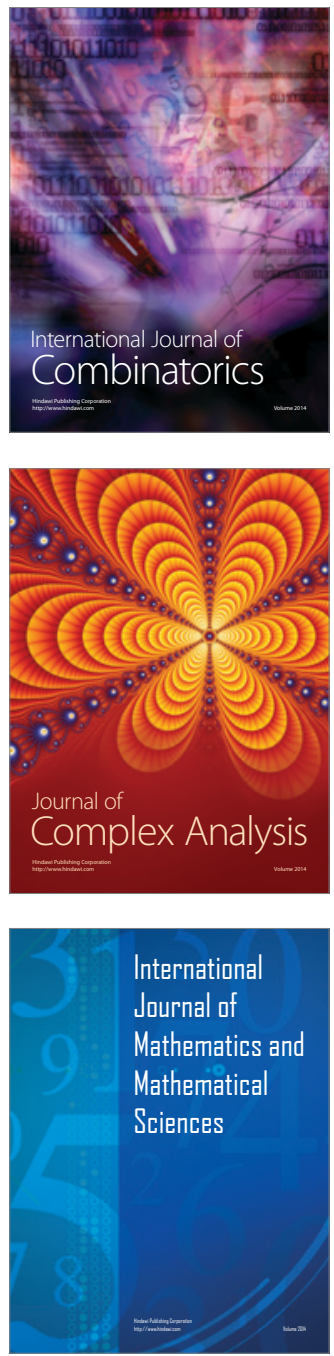
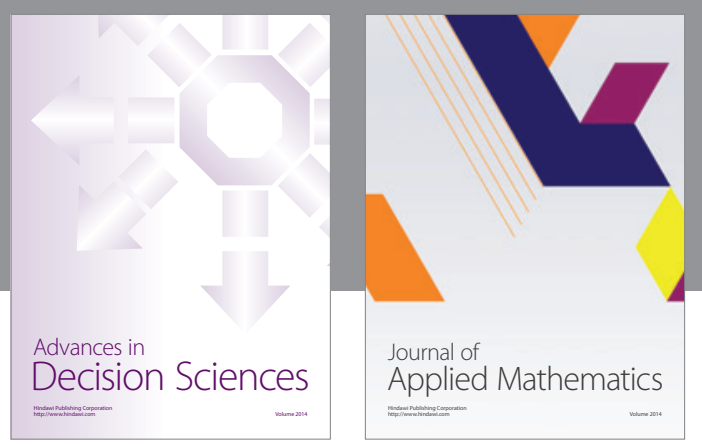

Algebra

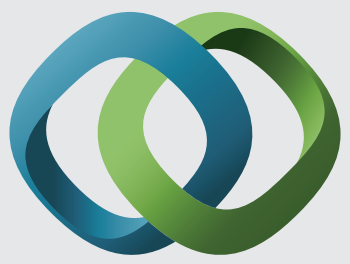

\section{Hindawi}

Submit your manuscripts at

https://www.hindawi.com
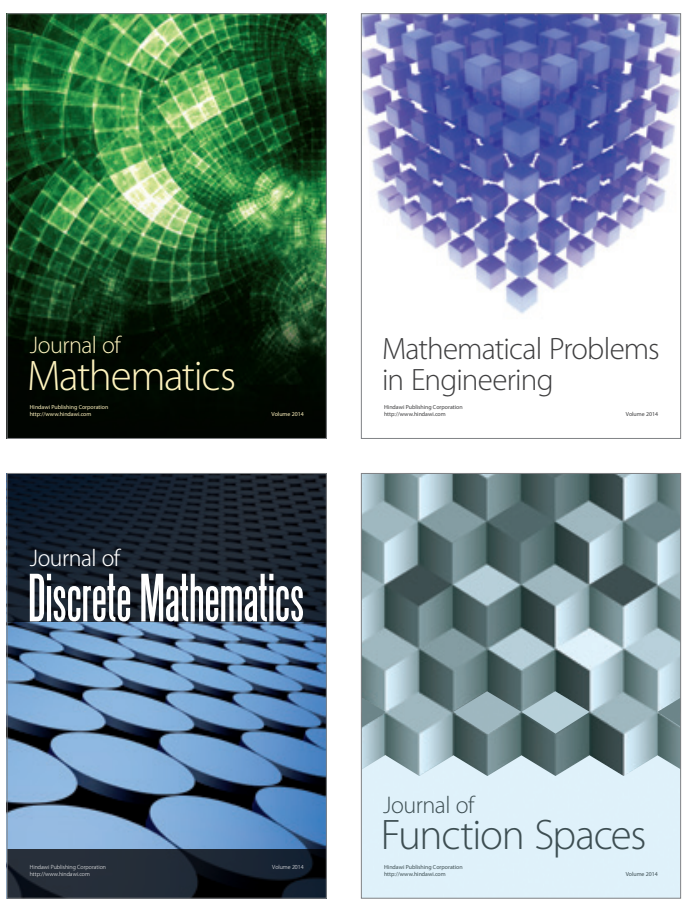

Mathematical Problems in Engineering
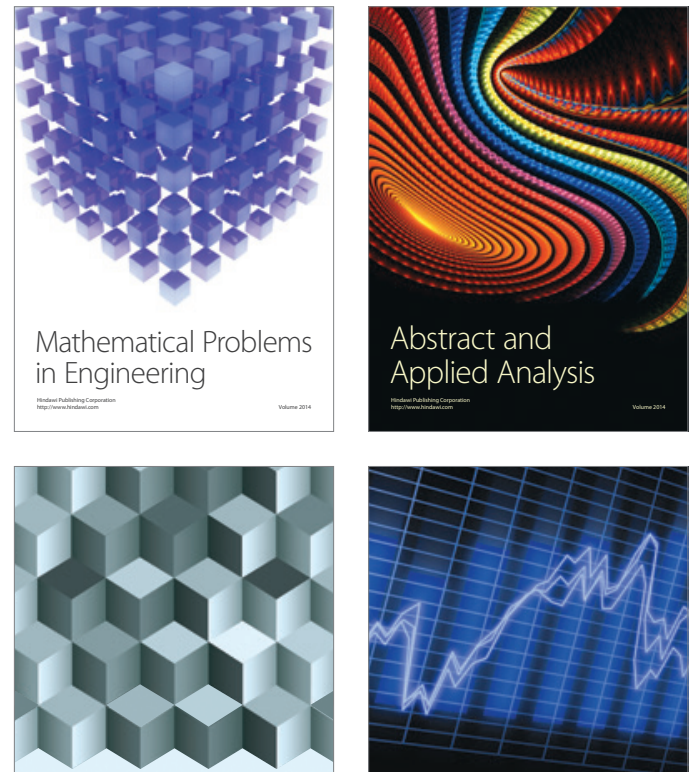

Journal of

Function Spaces

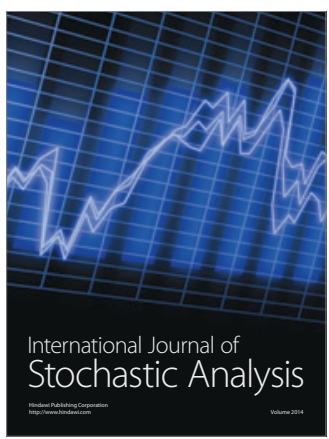

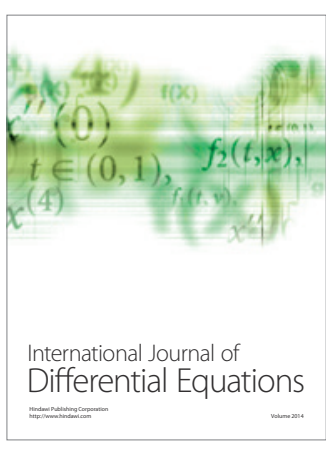
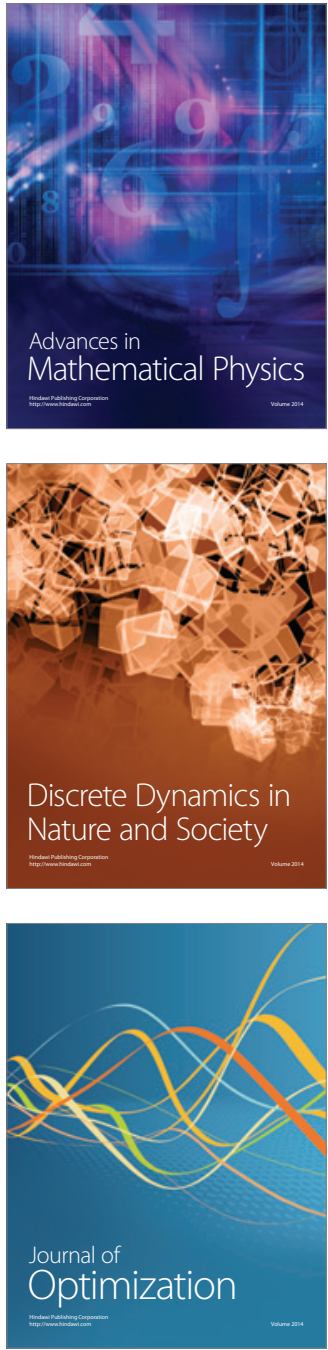